\title{
Gibbs Prior Models, Marching Cubes, and Deformable Models: A Hybrid Framework for 3D Medical Image Segmentation
}

\author{
Ting Chen ${ }^{1}$ and Dimitris Metaxas ${ }^{2}$ \\ 1 University of Pennsylvania, Philadelphia PA 19104, USA, \\ chenting@graphics.cis. upenn.edu, \\ 2 Rutgers University, Piscataway NJ 08854, USA
}

\begin{abstract}
Hybrid frameworks combining region-based and boundarybased segmentation methods have been used in 3D medical image segmentation applications. In this paper we propose a hybrid 3D segmentation framework which combines Gibbs models, marching cubes and deformable models. We use Gibbs models to create 3D binary masks of the object. Then we use the marching cubes method to initialize a deformable model based on the mask. The deformable model will fit to the object surface driven by the gradient information in the original image. The deformation result will then be used to update the parameters of Gibbs models. These methods will work recursively to achieve a final segmentation. By using the marching cubes method, we succeed in improving the accurancy and efficiency of $3 \mathrm{D}$ segmentation. We validate our method by comparing the segmentation result with expert manual segmentation, the results show that high quality segmentation can be achieved with computational efficiency.
\end{abstract}

\section{Introduction}

Computerized segmentation has been playing an increasingly important role in medical image analysis applications such as object reconstruction, motion capture, medical animation, remote operation, and myocardium dynamic analysis. Although modern imaging modalities provide exquisite imagery of the anatomy, precise segmentation of medical images continues to pose a challenge.

Classical segmentation methods such as boundary based methods [2] [3] and region based methods [1] [6] may lead to local minima during segmentation since they cannot combine the regional information and the boundary information of the object. In [4] [15], hybrid segmentation frameworks have been developed to improve the segmentation results by combining the strength of each of these two methods. In a hybrid framework, region-based methods and boundary-based methods work recursively to push each other out of local minima during segmentation. However, in those hybrid frameworks, those two segmentation methods are just loosely coupled: the boundary information provided by boundary-based methods cannot be used by region-based methods, which makes it difficult for region-based methods to get out of local minima. 
For the purpose of diagnosis and surgery planning, we need accurate segmentation methods for both $3 \mathrm{D}$ volumes and $2 \mathrm{D}$ slices. In [16], a level set method has been used to get 3D segmentation for brains. However, the segmentation process takes a long time (over 4 hours for one volume). In [14] a 3D model deforms to fit the object surface driven by the "balloon" force. The model expands like a balloon until its surface confronts the object surface. One weakness of the method is that the model cannot easily fit into object surface features such as concavities and convexities.

In this paper we extend our methodology in [7] into a 3D segmentation method by integrating Gibbs models [6], marching cubes[17] [18] and deformable models [11] into a hybrid framework.

Unlike hybrid frameworks in [4] [15], we use the deformable model segmentation to update the boundary parameters of the Gibbs prior model, which makes our method have a consistent performance without a training session. By using the marching cubes method to initialize deformable models close to the object surface, we skip the balloon-fitting process in [14]. This helps to greatly reduce the segmentation time for big 3D volumes and enables the deformable model fit into concavities and convexities.

To demonstrate the utility of the method in clinical applications, we apply the hybrid framework to brain (2 without lesion, 15 with lesion) and tumor segmentation in MR images. Our segmentation method is validated based on manual segmentation by experts. The results show over $90 \%$ similarity between the result of our hybrid method and the ground truth.

The paper is organized as follows: In section 2 we describe our methodology. In section 3, experiment results are given to demonstrate how to use our segmentation method. In section 4 we present validation results. In section 5 we give our conclusion.

\section{Methodology}

In this section we describe four internal modules of our hybrid segmentation framework and how they work sequentially to get the global segmentation result.

\subsection{High Order Gibbs Prior Models}

In [7] we proposed a high order Gibbs prior model that can use boundary information explicitly as well as the region information.

Using the Equivalence Theorem proved by Hammersley and Clifford [9] and the Bayesian framework, the segmentation problem can be degraded to the minimization of an energy function.

The energy function of our Gibbs prior model has the following form:

$$
H_{\text {prior }}(X)=H_{1}(X)+H_{2}(X)
$$

where $H_{1}(X)$ models the piecewise pixel homogeneity statistics, $H_{2}(X)$ models the continuity of the boundary. 
One important advantage of our high order Gibbs Prior model over other region-based methods is that it uses the boundary information of the object as well as the regional information. $H_{2}(X)$ has the following form:

$$
H_{2}(X)=\vartheta_{2} \sum_{s \in X} \sum_{i=1}^{N} W_{i}(s)
$$

where $s$ is a pixel, $\vartheta_{2}$ is the weight term for the boundary continuity, $N$ is the number of local configurations that may lie on the boundaries. $W_{i}(s)$ are potential functions defined on 3 by 3 clique with $s$ at center.

Assuming we assign "1" to pixels inside the object and "0" to pixels in the background, for a clique contains 3 by 3 pixels, there are altogether $2^{9}$ possible $(1,0)$ configurations. We group these configurations into six classes of cliques and assign different values to potential function of each class of clique. We assign smaller values to clique configures that locate at smooth and continuous boundaries. Therefore, when we minimize $H_{2}(X)$, the pixels in the image will alter their gray values to form clique configurations that have been assigned a low value. These alternations will lead to smooth and continuous boundaries in the resulting image. For more details about the Gibbs prior model, please refer to $[7][8]$.

To save time, we only use 2D Gibbs models in our framework. For 3D volumes, we apply $2 \mathrm{D}$ Gibbs models onto each slice in the volume to get a series of $2 \mathrm{D}$ binary masks of the object region. We combine these $2 \mathrm{D}$ binary masks to form a $3 \mathrm{D}$ binary mask as the input of the marching cubes method.

\subsection{Marching Cubes}

We use the discretized marching cubes method proposed by Montani in [18] to build a 3D mesh based on segmentation results of Gibbs models.

We construct the deformable surface by scanning through all the voxels in the 3D volume. Each voxel is a cube created from 8 pixels, four each from a slice. We give an index to the 13 potential locations for the nodes on the deformable model surface in this cube and define 13 possible plane incidences onto which elements of the deformable model can lie.

There are altogether 256 possible on-off combinations of the cube vertex. However, we only need to consider the 16 final cube configurations according to [18]. Those 256 vertex on-off combinations can be converted into one of the 16 configurations by a finite number of inverse, flip, and rotation operations without causing any topological ambiguity. We record the necessary operation sequence for each one of the 256 vertex combinations to convert to one of the final 16 configurations into a lookup table and the final configuration of each combination into another lookup table.

The size of these two lookup tables are both 256 bytes. They are small enough to be loaded in the memory before the element creation process begins

After initializing the lookup tables, we scan through the whole 3D image volume. We create elements in each voxel based on the final configuration of the 
current vertex combination according to second lookup table. Then we decide the coordination of nodes on the deformable surface by transferring the element using transfer sequence in the first lookup table. Since both lookup tables have already been loaded into the memory, the element construction process runs very fast (shown in table 2). Finally we combine elements together to form a $3 \mathrm{D}$ deformable mesh.

\subsection{Deformable Models}

We use Gibbs models and the marching cubes method to initialize a deformable surface that is close to the object surface. We write the deformable model dynamics in the form of the first order Lagrangian method:

$$
\dot{\mathbf{d}}+\mathbf{K d}=f_{\text {ext }}
$$

where $\dot{\mathbf{d}}=\frac{\partial \mathbf{X}}{\partial t} . \mathbf{K}$ is the stiffness matrix. $f_{\text {ext }}$ is the external force which is a combination of a second order derivative gradient vector flow and balloon force.

\subsection{Gibbs Model Reparameterization}

In the first iteration of the recursive hybrid framework, the parameters of Gibbs prior models are set to default values (please refer to section 2.1). To improve the segmentation result for Gibbs prior models, we update their parameters before restarting them in the following iterations.

Besides updating regional parameters such as the intensity and the standard deviation of the object, we also update the potential of the local configuration that may lead to different boundary conditions.

The clique potential of the higher order Gibbs Prior model should be proportional to the number of appearance of each type of cliques in the segmented binary image. For each clique, its potential is decided by:

$$
I_{h}(w)=\gamma \ln \left(\frac{r\left(t_{h}(w)\right)}{\# r\left(t_{h}(w)\right)}+1\right)
$$

where $t_{h}(w)$ is the clique type for the clique with pixel h at the center. $r\left(t_{h}(w)\right)$ is the number of occurrence of this clique type in the binary image. $\# r\left(t_{h}(w)\right)$ is the number of the configurations in the clique type.

Updated parameters represent a more accurate prior distribution than those of default values, which improves the performance of Gibbs models in the following iterations of the hybrid framework.

\subsection{Recursive Hybrid Framework}

Figure 1 shows internal modules and the data flow of 3D segmentation procedures. Gibbs models, the marching cubes method and the deformable model work recursively to get a global solution to the segmentation problem. 


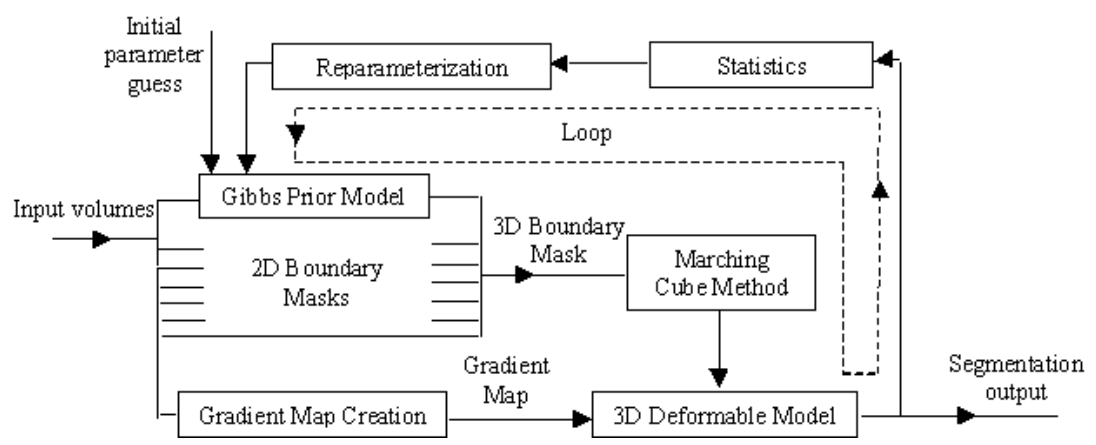

Fig. 1. Flow-Chart for 3D-segmentation hybrid framework.

\section{$3 \quad$ Experiments and Results}

We illustrate our hybrid segmentation framework by applying it onto a 3D MR image volume of a brain with a tumor region (Figure 2(a) shows one slice of the volume). The image volume size is 256 by 256 by 32 pixels. 32 2D Gibbs Prior models are used to create a 3D binary mask for the tumor region. We use the marching cube method to create a surface for the deformable model to begin with. The deformable model is driven by a second derivative gradient vector flow. The segmentation process stops after two iterations. Figure 2(b) shows the segmentation result of the Gibbs model applied on the slice using default parameter values. Figure 2(c) shows the segmentation result of the Gibbs model using parameter values updated based on the segmentation result of deformable model. In Figure 2(d) we show the final segmentation result of the hybrid framework. For quality evaluation, we overlay the segmentation result onto the original image as in Figure 2(e), the dark region in the image is the location of final our segmentation. We also show the initial deformable surface constructed using marching cube method in Figure 2(f), and the segmentation result after the deformation in Figure 2(g), 2(h). Notice that the segmentation result of the Gibbs model is improved by using updated parameters (Figure 2(b) $2(\mathrm{c})$ ). It is evident that our hybrid framework is capable of getting the segmentation process out of local minima. The fact that in Figure 2(g) and 2(h) the deformable model fits well at concavities and convexities proves that our hybrid framework has a good performance in segmenting complicate object surfaces. The total segmentation time is about 12 minutes for 2 iterations, which is much shorter than the method in [16].

We also run our method to segment the whole brain (white matter plus gray matter, provided by Peter Ratiu's group at Harvard University). The size of these MR volumes are 256 by 256 by 124 pixels. The processing time for one segmentation iteration is about 10 minutes. We show $3 \mathrm{D}$ segmentation results of 3 volumes and the corresponding intersections of those segmentation results on coronal planes in figure 3 and figure 4 . These experiments are done on a P4 $2 \mathrm{GHz}$ PC with $1 \mathrm{G}$ memory. The operating system is WinXP. 


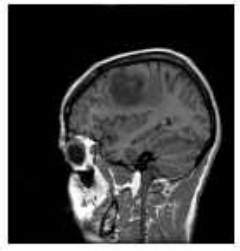

a

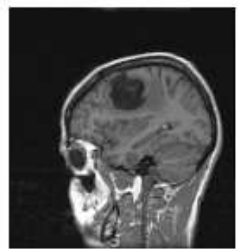

e

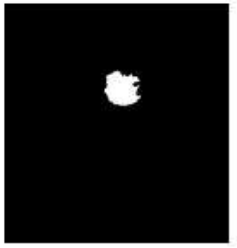

b

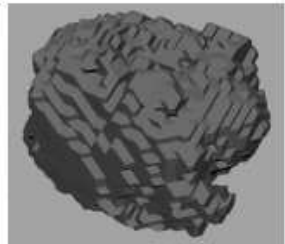

f

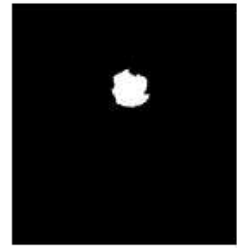

c

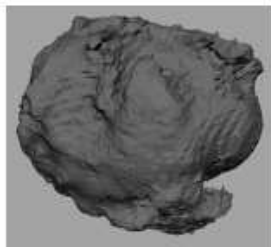

g

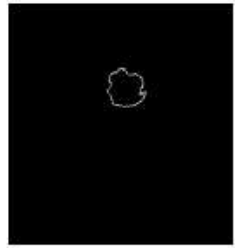

d

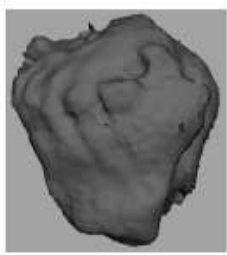

h

Fig. 2. Segmentation of a tumor in the brain from MR image, a. the original image; b, c. the Gibbs model segmentation result in the first and second iterations; d. the final segmentation result of the hybrid framework; e. the segmentation result overlays upon the original image; f. the initial deformable surface; g, h. 2 views of the final segmentation result in $3 \mathrm{D}$

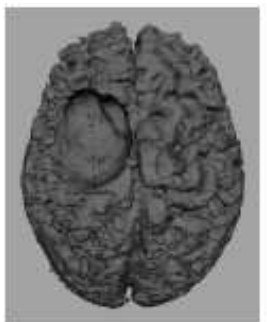

a

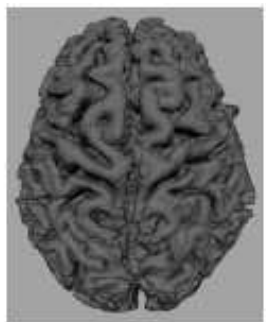

b

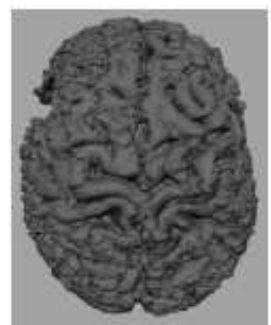

c

Fig. 3. Brain segmentation results. There are tumor regions on the brain surface in a) and c) and have been removed during segmentation

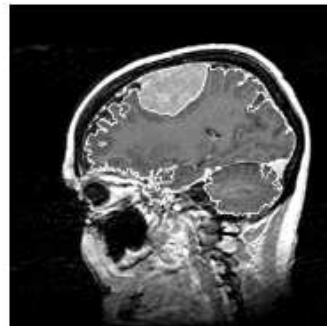

a

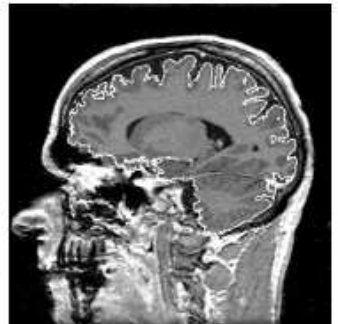

b

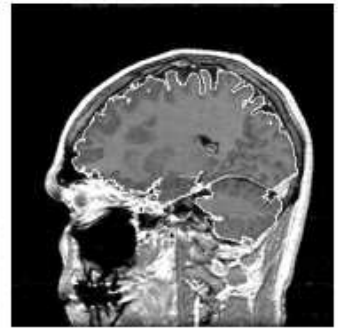

c

Fig. 4. Corresponding intersections of segmentation results in Figure 3) on coronal planes, the white lines are the final locations of deformable models 


\section{Validation}

We use the framework proposed by Chalana et al in [13] and Udupa in [12] for quantitative validation.

We compute the false negative volume fraction (FNVF), false positive volume fraction (FPVF) and positive volume fraction (TPVF) using experimental results and manual segmentations by experts to evaluate the validity of our segmentation framework quantitatively. Validation results based on segmentation of 10 brain tumor data are shown in table 1 .

All the results are in percentages. Hybrid segmentation results (in the third row) have a higher TPVF and lower FPVF than those of Gibbs prior models or deformable models alone, which shows the superiority of our hybrid method. In addition, the segmentation results of the Gibbs models (in the first and forth row) improve after using updated parameters, which proves that our method is capable of getting out of local minima when there is limited prior information available.

We also record the deformation time of some experiments to compare the performance of our method on different subjects. The results are in table 2 .

Table 1. Quantitative validation results.

\begin{tabular}{|r||c|c|c|}
\hline & FNVF & FPVF & TPVF \\
\hline \hline Gibbs Model (default parameters) & $8.7 \%$ & $5.5 \%$ & $91.3 \%$ \\
\hline Deformable Model & $5.9 \%$ & $6.5 \%$ & $95.1 \%$ \\
\hline Gibbs Model plus Deformable Model & $2.8 \%$ & $5.6 \%$ & $97.2 \%$ \\
\hline Gibbs Model (updated parameters) & $7.1 \%$ & $5.1 \%$ & $93.4 \%$ \\
\hline
\end{tabular}

Table 2. Deformable fitting time for some experiments. The tumor data size is 256 by 256 by 40 . The brain data size is 256 by 256 by 53 . The jaw data size is 256 by 256 by 102. The heart data size is 256 by 256 by 11 .

\begin{tabular}{|r||c|c|c|c|}
\hline & tumor & brain & jaw & heart \\
\hline \hline Initialization & 1 second & 1 second & 3 seconds & 1 second \\
\hline Construct Gradient Map & 3 seconds & 4 seconds & 11 seconds & 1 second \\
\hline Construct Mesh & 2 seconds & 5 seconds & 13 seconds & 1 second \\
\hline Deformable Fitting & 25 seconds & 71 seconds & 75 seconds & 6 seconds \\
\hline
\end{tabular}

\section{Conclusion}

This paper has presented a 3D hybrid segmentation framework with high accurancy and efficiency. The Gibbs model and the deformable model are better integrated by the use of the marching cubes method and the reparameterization module. The method does not need a training session and has a strong performance in segmenting complicate objects in 3D image volumes with low SNR.

Acknowledgement. The work presented in this paper is a part of the itk project sponsored by NIH/NLM. 


\section{References}

1. D. H. Ballard, C. M. Brown.: Computer vision. Prentice Hall, (1982)

2. M. Kass, A. Witkin, and D. Trerzopoulos.: Snakes: Active contour models. Intl. J. of Computer Vision. 1(4) (1988) 321-331

3. M. Worring, A. W. M. Smeulders, L. H. Staib, and J. S. Duncan.: Parameterized feasible boundaries in gradient vector fields. Computer Vision and Image Understanding. 63(1) (1996) 135-144

4. T. N. Jones, D. N. Metaxas.: Automated 3D segmentation using deformable models and fuzzy affinity. In J. Duncan and G. Gindi, editors, Lecture notes in computer Science. Volume 1230: IPMI '97 113-126. Springer, 1997

5. M. T. Chan, G. T. Herman and Emanuel Levitan.: A Bayesian Approach to PET Reconstruction Using Image-Modeling Gibbs Prior. IEEE Transaction on Nuclear Science, Vol. 44, No. 3, June 1997

6. S. Geman, D. Geman.: Stochastic relaxation, Gibbs distributions, and the Bayesian restoration of images. IEEE Trans. Pattern Anal. Mach. Intell. 6 (1984) 721-741

7. T. Chen, D. Metaxas.: Image Segmentation based on the Integration of Markov Random Fields and Deformable models. MICCAI (2000)

8. Gerhand Winkler.: Image Analysis, random Fields and Dynamic Monte Carlo Methods, (1995) Springer

9. J. M. Hammersley, P. Clifford.: Markov fields on finite graphs and lattices. Preprint University of California, Berkeley

10. C. Xu, D. L. Pham, and J. L. Prince.: Medical Image Segmentation Using Deformable Models, Handbook of Medical Imaging Volume 2: Medical Image Processing and Analysis. page 129-174, edited by J.M. Fitzpatrick and M. Sonka, SPIE Press, May (2000)

11. D.N. Metaxas.: Physics-Based Deformable Models: Application to Computer Vision, Graphics and Medical Imaging. (1996)

12. J. K. Udupa, V. R LeBlanc, H. Schmidt, C. Imielinska, P. K. Saha, G. J. Grevera, Y. Zhuge, L. M. Currie, P. Monholt and Y. Jin.: A Methology for Evaluating Image Segmentation Algorithms. In Proceedingss of SPIE: Medical Imaging, San Diego, CA, 4684, (2002) 266-277

13. Vikram Chalana, Yongmin Kim.: A Methodology for Evaluation of Boundary Detection Algorithms on Medical Images. IEEE Transaction on Medical Imaging. volume 16, (1997) 642-652

14. L. D. Cohen, I. Cohen.: Finite Element Methods for Active Contour Models and Balloons for 2D and 3D Images. IEEE Transaction on Pattern Analysis and Machine Intelligence 15(11): 1131-1147

15. A. Chakraborty, J. S. Duncan.: Integration of Boundary Finding and Region-based Segmentation Using Game Theory. Information Processing in Medical Imaging: Proceedings of the 14th International Conference (IPMI'95), Ile de Berder, France, June, 1995

16. C. Xu, D. L. Pham, M. E. Rettmann, D. N. Yu, and J. L. Prince.: Reconstruction of the Human Cerebral Cortex from Magnetic Resonance Images. IEEE Transactions on Medical Imaging, 18(6), pp. 467-480, June, 1999

17. William E. Lorenson, Harvey E. Cline.: Marching Cubes: A High-resolution 3D Surface Construction Algorithm. Proceedings of the 14th annual conference on Computer graphics and interactive techniques., 163-169

18. C. Montani, R. Scateni and R. Scopigno.: Discretized Marching Cubes. In Proceedings of the Visualization '94 Congress, (1994) 281-287 